\title{
Neutron-antineutron transition as a test-bed for dynamical CPT violations
}

\author{
Andrea Addazi \\ Dipartimento di Fisica, Università di L'Aquila, 67010 Coppito AQ and LNGS, \\ Laboratori Nazionali del Gran Sasso, 67010 Assergi AQ, Italy
}

(Dated: May 16, 2022)

\begin{abstract}
We show a simple mechanism for a dynamical CPT violation in the neutron sector. In particular, we show a CPT-violating see-saw mechanism, generating a Majorana mass and a CPT violating mass for the neutron. CPT-violating see-saw involves a sterile partner of the neutron, living in a hidden sector, in which CPT is spontaneously broken. In particular, neutrons (antineutrons) can communicate with the hidden sector through non-perturbative quantum gravity effects called exotic instantons. Exotic instantons dynamically break R-parity, generating one effective vertex between the neutron and its sterile partner. In this way, we show how a small CPT violating mass term for the neutron is naturally generated. This model can be tested in the next generation of experiments in neutron-antineutron physics. This strongly motivates researches of CPT-violating effects in neutron-antineutron physics, as a test-bed for dynamical CPT-violations in SM.
\end{abstract}

PACS numbers: 11.25.Wx,11.30.Er,11.30.Fs,14.20.Dh

Keywords: Neutron-Antineutron, Strings, Baryon violations, CPT violations

\section{INTRODUCTION}

Is CPT a fundamental symmetry of Nature?

Only experimental observables can answer to this crucial question. CPT is strictly connected with other deep issues like Lorentz invariance (LI), Causality and Locality. For this motivation, CPT seems an untouchable symmetry of Quantum Field Theories (QFT). However, it is commonly retained that QFT are effective theories of a more fundamental one, including quantum gravity. In this generic idea, locality or LI could be emergent/approximated principles rather than fundamental ones [24]. On the other hand, CPT could be spontaneously or dynamically broken even if starting from a CPT-preserving theory. For example, one can envisage the presence of a hidden sector, in which CPT is spontaneously broken. In this case, mediators can transmit informations of CPT-violations (CPTV) from the hidden sector to Standard Model (SM). CPTV can manifest itself in particle-antiparticle mass differences (even if it is not the only CPTV observable, in general). For general papers and reviews on CPT-violations, see [1. Kaons (Antikaons) are particularly sensitive to CPTV effects, so that the actual limits are very stringent: $R_{K}=\left|\Delta m_{K \bar{K}}\right| / m_{K}<8 \times 10^{-19}$ [2]. On the other hand, limits on neutron (antineutron) are much milder than the previous ones: only $R_{n}=\left|\Delta m_{n \bar{n}}\right| / m_{n}<(9 \pm 5) \times 10^{-5}$ [3]. Next generation of experiments will improve limits on neutron-antineutron physics [4]. In [5], it was proposed that if a neutron-antineutron transition was found, it would be a test for CPT. See also [6] for a recent paper on CPTV in $n-\bar{n}$ transitions.

In this paper, we propose a simple mechanism, dynamically breaking CPT in SM sector, generating a CPT violating mass term only for the neutron! This mechanism can avoid stringent limits on kaons as well as for other possible channels. We propose a CPTV see-saw mechanism for the neutron [25], in which a sterile part-

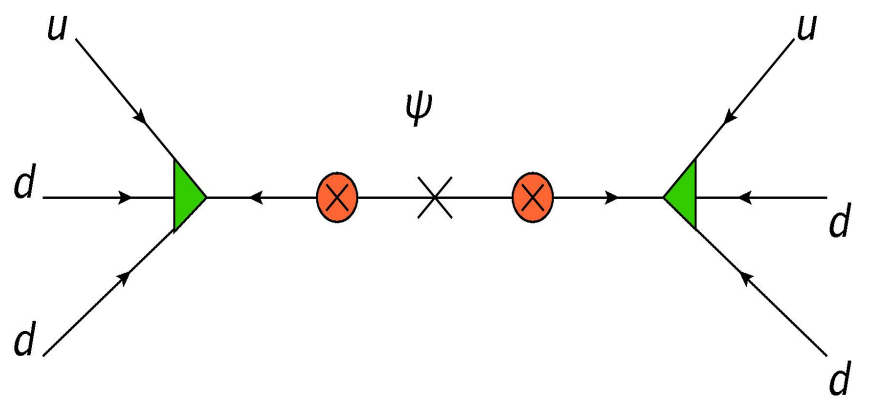

FIG. 1: Diagram of a CPT-violating see-saw mechanism for the neutron. $u, d, d$ are right-handed up and down quarks, while $\psi$ is a partner of the neutron, named "neutronic weight" or briefly "nweight". Black cross is a Majorana mass for $\psi$, while red-crosses are CPTV mass terms for $\psi$. As a consequence, $\psi, \bar{\psi}$ get a CPT violating mass matrix. As a CPTV see-saw mechanism, also a CPT violating mass matrix for $n, \bar{n}$ is generated. The 'green' vertices can be directly generated by Exotic Instantons.

ner of the neutron, called "neutronic weight" or briefly "nweight", living in a "CPTV hidden sector", can generate a Majorana mass and a CPT violating mass for the neutron. In a "CPTV hidden sector", we assume that $\mathrm{CPT}$ is violated by an unspecified mechanism, generating an unsuppressed CPTV mass for the nweight. Nonperturbative stringy effects, called exotic instantons, can provide privileged portals from the hidden sector to neutrons. For these motivations, a CPTV in neutron physics seems strongly sustained by (exotic) instantons-mediated models.

\section{CPTV SEE-SAW MECHANISM FOR THE NEUTRON}

Let us introduce the basic idea of our model. We will justify our assumptions later. We introduce a fermionic 
field $\psi$ living in a hidden sector. We call such a fermion "neutronic weight" or "nweight". Such a particle is a singlet with respect to SM gauge group (but not necessary for other hidden gauge groups), i.e $\psi(1,1,0)$ of $S U(3) \times S U(2) \times U(1)$. We assume that $\psi$ has a Majorana and a Dirac Mass term, $m_{\psi} \bar{\psi} \psi+\mu_{\psi} \psi^{2}+$ h.c. However, $\psi$ has also a large CPT-violating mass term $\Lambda_{C P T V} \psi^{\dagger} \psi+$ h.c. Such a mass term is associated to a local Lorentz symmetry breaking in the hidden sector:

$$
a_{\mu} \bar{\psi} \gamma^{\mu} \psi \rightarrow \Lambda_{C P T V} \psi^{\dagger} \psi
$$

where $a_{\mu}$ is a spurion vector field getting an expectation value $\left\langle a_{0}\right\rangle$ so that $\left\langle a_{0}\right\rangle \bar{\psi} \gamma_{0} \psi=\Lambda_{C P T V} \psi^{\dagger} \psi$. At this point, one could be 'afraid' about the fact that such a term seems gauge equivalent to zero such as an electric potential. However, in a multifermionic theory for the hidden sector such a conclusion is in general not true, as discussed in litterature cited above [26- 27.

Now, let us consider the following effective interactions

$$
\mathcal{L}_{\text {eff }}=\mathcal{L}_{1}+\mathcal{L}_{2}
$$

where

$$
\begin{gathered}
\mathcal{L}_{1}=\mu_{n \psi} n \psi+h . c \\
\mathcal{L}_{2}=m_{\psi} \bar{\psi} \psi+\mu_{\psi} \psi \psi+\Lambda_{C P T V} \psi^{\dagger} \psi+h . c
\end{gathered}
$$

$\mathcal{L}_{1}$ is an effective vertex of the neutron and the nweight field, as a portal to the hidden sector. We will discuss later how can be possible the generation of such an effective vertex, without generating other dangerous vertices. Lagrangian $\mathcal{L}_{\text {eff }}$ can give rise to a $\mathrm{CPT}$ violating mass term $\mu_{C P T V} n^{\dagger} n+$ h.c. This is a simple example of a CPT-violating see-saw mechanism for the neutron. The associated diagram is shown in Fig.1. In particular, $\psi, \bar{\psi}$ have a non-diagonal mass matrix

$$
\mathcal{M}_{\psi \bar{\psi}}=\left(\begin{array}{cc}
m_{\psi}+\Lambda_{C P T V} & \mu_{\psi} \\
\mu_{\psi}^{*} & m_{\psi}-\Lambda_{C P T V}
\end{array}\right)
$$

On the other hand, this is a sub-matrix of neutronnweight system. In basis $(n, \bar{n}, \psi, \bar{\psi})$, the complete system is

$$
\mathcal{M}_{C P T V}=\left(\begin{array}{ll}
\mathcal{M}_{n \bar{n}}^{0} & \mathcal{M}_{n \psi} \\
\mathcal{M}_{n \psi}^{\dagger} & \mathcal{M}_{\psi \bar{\psi}}
\end{array}\right)
$$

with

$$
\mathcal{M}_{n \psi}=\left(\begin{array}{cc}
\mu_{n \psi} & 0 \\
0 & \mu_{n \psi}^{*}
\end{array}\right)
$$

and $\mathcal{M}_{n \bar{n}}^{0}=m_{n} \mathcal{I}_{2 \times 2}$.

The general CPTV see-saw formula for the neutron is

$$
\mathcal{M}_{n \bar{n}}=-\mathcal{M}_{n \psi}^{\dagger} \mathcal{M}_{\psi \bar{\psi}}^{-1} \mathcal{M}_{n \psi}
$$

Let us note that if $\Lambda_{C P T V}=m_{\psi}=0$, we recover the $\mathrm{CPT}$ preserving case with a Majorana nweight. In this

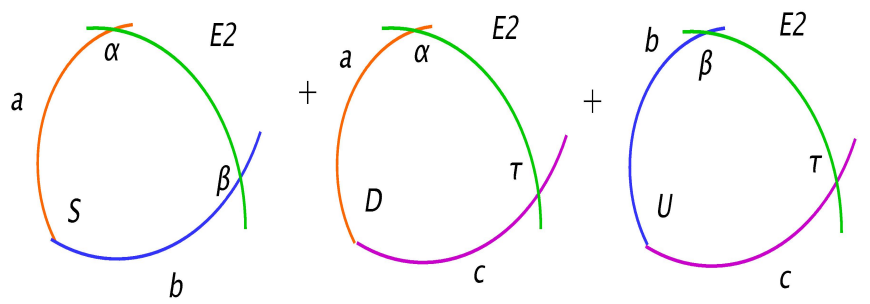

FIG. 2: Mixed disk amplitudes generating an effective $n-\psi$ vertex. The E2-instanton is in green, and it intersects ordinary D6-branes' stacks. This generates the desired effective interactions among ordinary fields and modulini.

case, $\left|m_{n}-m_{\bar{n}}\right|=0$, but a Majorana mass for the neutron (antineutron) is generated. Assuming $\mu_{\psi}>>m_{n}, \mu_{n \bar{n}} \simeq$ $\mu_{n \psi}^{2} / \mu_{\psi}$. On the other hand, if nweight has also a Dirac mass term, but again in CPT-preserving phase $\Lambda_{C P T V}=$ 0 , we obtain a Majorana mass $\mu_{n \bar{n}}=-\mu_{n \psi}^{2} \mu_{\psi} /\left(m_{\psi}^{2}-\mu_{\psi}^{2}\right)$. Now, let us consider CPTV case $\Lambda_{C P T V} \neq 0$. In this case, not only the neutron Majorana mass is non-trivially affected, but we also obtain a difference in neutron and antineutron Dirac masses:

$$
\begin{gathered}
\mu_{n \bar{n}}=-\frac{\mu_{n \psi}^{2} \mu_{\psi}}{-\Lambda_{C P T V}^{2}+m_{\psi}^{2}-\mu_{\psi}^{2}} \\
\left|m_{n}-m_{\bar{n}}\right|=\frac{2 \mu_{n \psi}^{2} \Lambda_{C P T V}}{\left|\Lambda_{C P T V}^{2}-m_{\psi}^{2}+\mu_{\psi}^{2}\right|}
\end{gathered}
$$

\section{THEORETICAL INTERPRETATIONS OF THE CPTV-SEESAW MODEL.}

The main theoretical problems behind our toy-model are: why does such a singlet field interact only with neutrons? Can such a field destabilize nuclei? Can CPTV phases be transmitted also in kaons or other well constrained channels?

In this section, we will comment how possible can be a situation in which $\psi$ has one and only one portal-operator like $\mathcal{O}_{n \psi}=\psi u^{c} d^{c} d^{c} / \Lambda_{n \psi}^{2}$, without generating a plethora of other dangerous operators. In other words, we desire a mechanism to dynamically break baryon number without generating all possible baryon and lepton violating operators. As shown in [7, 8], $(B-L)$-parity can be dynamically broken from non-perturbative quantum gravity effects known as exotic stringy instantons. This class of instantons corresponds to Euclidean D-branes (or E-branes), intersecting physical D-brane stacks. More precisely, in IIA string-theory [28], the class of "exotic instantons" corresponds to E2-branes wrapping different 3 -cycles on the Calabi-Yau compactification with respect to ordinary D6-branes [29.

In intersecting D-branes' models, Standard Model content and the extra singlet can be easily reconstruct in the low energy limit. On the other hand, an E2instanton, with the appropriate intersections and Chan- 
Paton group, can generate a superpotential like $\mathcal{W}_{n \psi}=$ $S U^{c} D^{c} D^{c} / \mathcal{M}_{0}$ where $\mathcal{M}_{0}=e^{+S_{E 2}} M_{S}$, where $M_{S}$ is the string scale and $e^{-S_{E 2}}$ is a function of geometric moduli associated to 3 -cycles of E2-brane on $C Y_{3}$. Now, $S$ is a nweight superfield with $\psi$ as the lowest component. These superpotential terms generate operator $\mathcal{O}_{n \psi}=\psi u^{c} d^{c} d^{c} / \Lambda_{n \psi}^{2}$ with $\Lambda_{n \psi}^{2}=\mathcal{M}_{0} m_{\tilde{g}}$ ( $m_{\tilde{g}}$ gaugino mass, like gluino, photino or zino).

A $\mathcal{W}_{n \psi}$ can be obtained in a D-brane model with extra vector-like pairs of color-triplets, through the generation of a non-perturbative mass term for these, as described in our papers cited above. But here, we would like to suggest another possibility: $\mathcal{W}_{n \psi}$ can be directly generated from exotic instantons, without the needing of coloredmediators! In Fig.2, we show an E2-instanton directly generating the neutron-nweight portal, through mixed disk amplitudes. In particular: $D^{c}$ comes from excitations of open strings attached to one $U(1)$-stack (named a) and a $U(3)_{c}$-stack (named c); $U^{c}$ from $U(1)^{\prime}$-stack (named b) and $U(3)_{c}$-stack; $S$ from $U(1)$-stack (a) and $U^{\prime}(1)$-stack (b). Let us remind that we are considering stacks of D6-branes wrapping 3-cycles on $C Y_{3}$. The E2brane intersects three times the $\mathbf{a}$, two times the $\mathbf{b}$ and one time the c. Effective interactions among ordinary superfields $S, U^{c}, D^{c}$ and modulini $\alpha^{f=1,2,3}, \beta^{g=1,2}, \tau^{i=1,2,3}$ (living between ordinary D6-branes and $E 2$-branes):

$$
\mathcal{L}_{E 2} \sim Y_{f g}^{(1)} S \alpha^{f} \beta^{g}+Y_{f}^{(2)} \alpha^{f} D_{i}^{c} \tau^{i}+Y_{g}^{(3)} \beta^{g} U_{i}^{c} \tau^{i}
$$

where $i$ is the color index of $U_{c}(3), f=1,2,3$ labels the number of $\alpha, g=1,2$ the number of $\beta ; Y^{(1,2,3)}$ are Yukawa matrices coming from mixed disk correlators. Integrating-out modulini

$$
\int d^{3} \tau d^{3} \alpha d^{2} \beta e^{\left\{Y_{f g}^{(1)} S \alpha^{f} \beta^{g}+Y_{f}^{(2)} \alpha^{f} D_{i}^{c} \tau^{i}+Y_{g}^{(3)} \beta^{g} U_{i}^{c} \tau^{i}\right\}}
$$

we obtain the desired superpotential, with $\mathcal{M}_{0}=$ $M_{S} e^{+S_{E 2}}$. Such a mechanism can be embedded in Dbrane models like $U_{3}(3) \times U_{L}(2) \times U(1) \times U(1)^{\prime} \times G$ or $U_{3}(3) \times S p_{L}(2) \times U(1) \times U(1)^{\prime} \times G$, where $G$ is a generic gauge extension obtained by the global D-brane construction [30]. A complete classification of all consistent quivers is beyond the purpose of this short paper. Finally, let us comment that a CPTV mass term for $\psi$ can be introduced in our susy model, as a soft susy/CPT breaking parameter by "diagonal" R-R or NS-NS stringy fluxes 31.

\section{A. Proton is stable and CPTV in $K_{0}-\bar{K}_{0}$ are smaller than in $n-\bar{n}$}

Now let us comment two important phenomenological aspects of our model. First, a $\Delta B=1$ superpotential like $U^{c} D^{c} D^{c} S / \mathcal{M}_{0}$ could be dangerous: why not introduce other $\Delta B, \Delta L=1$ interactions between $S$ and $\mathrm{SM}$, immediately destabilizing the proton? The answer is because our mechanism has dynamically broken an initial $R$-parity, generating one and only one superpotential $U^{c} D^{c} D^{c} S / \mathcal{M}_{0}$. Other ones are not generated. See also our recent papers cited above for discussions on these aspects. This one, alone, cannot destabilize the proton. Let us note that also other discrete symmetries in the hidden sector can be introduced: in this case the exotic instanton dynamically breaks also these ones. In other words, $S$ not interacts with SM at perturbative level (we have defined it a hidden particle for this motivation). However, it can interact non-pertubatively through the E2-instanton considered.

Another comment regards kaons: one can construct a diagram generating a kaon-antikaon oscillation from $\mathcal{W}_{n \psi}=U^{c} D^{c} D^{c} S / \mathcal{M}_{0}$. In fact, calling $\tilde{s}$ the susy scalar partner of $\psi$, we can obtain an operator $\tilde{u}^{c} d^{c} d^{c} \tilde{s} / \mathcal{M}_{0}$, while $\tilde{s}$ can have a susy soft mass $m_{s}^{2} \tilde{s}^{\dagger} \tilde{s}+$ h.c. For our construction, $u, d$ can be up/down-like quarks, but they can have different flavors ( $c, s, t, b$ quarks). From these, we can obtain a D-term like diagram for $K_{0}-\bar{K}_{0}$ and in general a plethora of neutral meson oscillations. In our model set-up, it seems that CPTV phases propagate also in these channels. However, let us note that, these diagrams can be very suppressed with respect to $n-\bar{n}$ one: i) these have an extra one-loop suppression from integration of one squark and one $\tilde{s}$; ii) $m_{s}$ is a free-parameter that in principle can be putted also up to the Planck scale! iii) Stringy mixed disk amplitudes are not necessary democratic with flavor: coefficients $Y^{(1,2,3)}$ are Yukawa matrices with flavors. For these motivations, even if measures in mesons physics remained motivated in our model, they are expected to be strongly suppressed with respect to neutron ones!

\section{FURTHER IMPLICATIONS IN $n-\bar{n}$}

In this section, we will comment previous results, with possible implications in next generation of experiments on $n-\bar{n}$-transitions.

First, let us note that expression (9) implies

$$
R_{C P T V / C P T P}=\frac{\mu_{n \bar{n}}^{C P T V}}{\mu_{n \bar{n}}^{C P T P}}=\frac{m_{\psi}^{2}-\mu_{\psi}^{2}}{-\Lambda_{C P T V}^{2}+m_{\psi}^{2}-\mu_{\psi}^{2}}
$$

If $\Lambda_{C P T V}<<\left|\mu_{\psi}-m_{\phi}\right|, R_{C P T V / C P T P} \simeq 1: \mathrm{CPTV}$ in neutron-antineutron transition are strongly suppressed.

Let us consider a more interesting regime: $\Lambda_{C P T V}>>$ $\left|\mu_{\psi}-m_{\psi}\right|$. Usually a neutron-antineutron experiment is done in condition of a strongly suppressed magnetic field. But in condition of $\Delta m_{n \bar{n}} \neq 0$, it is more appropriate to test a $n-\bar{n}$ transition with $\left|\mu_{n} B\right| \simeq \Delta m_{n \bar{n}}$. In fact, in this case the transition probability is resonantly enhanced rather than suppressed [11. For example let us suppose that $\Delta m_{n \bar{n}} \simeq 10^{-14} \div 10^{-6} \mathrm{eV}$ : this case corresponds to a range of external magnetic fields $|B| \simeq$ $10^{-3} \div 10^{5}$ Gauss. Clearly, a test with very high magnetic fields up to $1 \div 10$ Tesla is technologically challenging, but 
a test with $0.1 \div 1$ Gauss seems simpler to be realized. From 10 , we can estimate that $\Delta m_{n \bar{n}} \simeq 2 \mu_{n \psi}^{2} / \Lambda_{C P T V}$, that in our instanton-mediated model corresponds to $2 \Lambda_{Q C D}^{6} / \Lambda_{N P}^{5}$, where $\Lambda_{N P}^{5}=\left(\mathcal{M}_{0}^{2} m_{\tilde{g}}^{2} \Lambda_{C P T V}\right) \simeq 0.1 \div$ $10 \mathrm{TeV}$. This scale is easy to obtain under several reasonable choices of parameters. For example, $\mathcal{M}_{0} \simeq$ $10^{3} \mathrm{TeV}, m_{\tilde{g}} \simeq 1 \mathrm{TeV}, \Lambda_{C P T V} \simeq 0.1 \div 10 \mathrm{MeV}$. In this case, we can marriage our scenario with a low string scale scenario $M_{S} \simeq 1000 \mathrm{TeV}$, with gaugini reachable at LHC. Otherwise, another possible scenario can be $\mathcal{M}_{0} \simeq 10^{3} \mathrm{TeV}, m_{\tilde{g}} \simeq 10^{3} \mathrm{TeV}, \Lambda_{C P T V} \simeq 0.1 \div 10 \mathrm{eV}$. Clearly, in these cases $\psi$ has small Lorentz Invariant masses $\left|m_{\psi}-\mu_{\psi}\right|<<10^{-1} \div 10^{7} \mathrm{eV}$. Let us note for example that for $\mu_{\psi} \simeq 10^{-19} \div 10^{-9} \Lambda_{C P T V}$, one can obtain an intriguing situation for $n-\bar{n}$ transitions in presence of external magnetic fields: $\mu_{n \bar{n}} \simeq 10^{-23 \div 25} \mathrm{eV}$ and $\Delta m_{n \bar{n}} \simeq 10^{9 \div 19} \mu_{n \bar{n}}$. However, a so light $\psi$ seems dangerous: it can lead to meson decays like $K^{0} \rightarrow \psi \bar{\psi}, \psi \psi$. These decays can be obtain with a one loop diagram of squarks. However, such a decay has a very suppressed rate for $\Lambda_{C P T V} \simeq 1 \mathrm{eV} \div 1 \mathrm{MeV}: \operatorname{Br}\left(K_{S(L)} \rightarrow \psi \bar{\psi}\right) \sim$ $\lambda_{\psi}^{2} / m_{K_{S, L}}^{2}\left(\Lambda_{Q C D} / \Lambda_{n \psi}\right)^{2} \mathcal{S}<10^{-31} \div 10^{-17}$, where $\lambda_{\psi}$ is the mass eigenvalue of $\psi$, and $\mathcal{S}$ represents other understood suppressions in our one-loop diagrams. The current limits extracted from existing data are only $\operatorname{Br}\left(K_{S(L)} \rightarrow\right.$ invisible $)<1.1 \times 10^{-4}\left(6.3 \times 10^{-4}\right)[12$. These limits are planned to be improved by $2-3$ orders of magnitude. In particular the planned experimental limits The planned experimental limits on $K \rightarrow$ invisible are expected to be at the level of $B\left(K_{S(L)} \rightarrow\right.$ invisible $)<$ $10^{-8}\left(10^{-6}\right)$ or below, comparable with other possible meson decays into invisible channels 12 . On the other hand, possible double decays $n n \rightarrow \psi \psi$ are automatically strongly suppressed: $\Gamma_{n n \rightarrow \psi \psi} \sim\left(\rho_{N} / m_{n}^{2}\right)\left(\Lambda_{Q C D} / \Lambda_{n \psi}\right)^{12}$ with $\rho_{N} \simeq 0.23 \mathrm{fm}^{-3}$, corresponding to $\tau_{n n \rightarrow \psi \psi} \sim 2 \times$ $10^{45} \mathrm{yr}\left(\Lambda_{\mathrm{n} \psi} / \mathrm{TeV}\right)^{12}$. A possible nuclei destabilization decays from $n \rightarrow \psi$ is automatically avoided by energy conservation. A transition $n \rightarrow \bar{n}$ in nuclei is strongly suppressed by the nuclear binding energy $V \simeq 10 \div 100 \mathrm{MeV}$. However, according to the analysis done in [13], a case in which $\Lambda_{C P Y}>>\mu_{\psi}, m_{\psi}$ leads to instabilities in the hidden sector 32. However, the analysis done in 13 can be avoid by string-inspired spontaneously or dynamically mechanisms, involving higher order self-interaction couplings, as specified in the same paper. For example, higher-derivatives can relevantly modify the dispersion relation of the nweight in the high energy limit while they are completely irrelevant in low energy limits. Such an issue also involves deep principles like non-locality. To interpret such an effective low energy model in a consistent framework will deserve future investigations.

In the following part, we will focus on more natural models, compatible with bounds of 13 .

Let us suppose that $m_{\psi}<<\Lambda_{C P T V} \simeq \mu_{\psi}$, in condition of suppressed magnetic fields. This case is free by instabilities, according to the analysis done in [13. In this case, $R_{C P T V / C P T P} \simeq 1 / 2$. This also implies a $\left|\Delta m_{n \bar{n}}\right| \simeq \mu_{\psi n}^{2} / \Lambda_{C P T V}$. On the other hand, an in- sightful way to rewrite these expressions is in term of $\mu_{n \bar{n}} /\left|\Delta m_{n \bar{n}}\right|$ :

$$
\frac{\mu_{n \bar{n}}}{\left|\Delta m_{n \bar{n}}\right|}=\frac{\mu_{\psi}}{2 \Lambda_{C P T V}}
$$

As a consequence, a situation in which $\Delta m_{n \bar{n}} \simeq \mu_{n \bar{n}}$ corresponds to $\Lambda_{C P T V} \simeq \mu_{\psi} / 2$. The actual limit on $\mu_{n \bar{n}}$ corresponds to $10^{-23} \mathrm{eV} \cdot \mu_{\psi n}=\Lambda_{Q C D}^{3} / \Lambda_{n \psi}^{2}$, where $\Lambda_{n \psi}$ is the New Physics scale in which such a $n-\psi$ vertex is generated, As a consequence $\mu_{n \bar{n}} \simeq \Lambda_{Q C D}^{6} /\left(3 \Lambda_{n \psi}^{4} \Lambda_{C P T V}\right)$, under the assumption $m_{\psi}<<\Lambda_{C P T V} \simeq \mu_{\psi}$. So, $\mu_{n \bar{n}}<$ $10^{-23} \mathrm{eV}$ corresponds to $\Lambda_{n \psi}^{4} \Lambda_{C P T V}>(100 \mathrm{TeV})^{5}$. The next generation of experiments will test $\Lambda_{n \psi}^{4} \Lambda_{C P T V} \simeq$ $(1000 \mathrm{TeV} / 3)^{5}$ scale. A possible interesting scenario can be $\Lambda_{n \psi} \simeq \Lambda_{C P T V} \simeq \mu_{\psi} / 2 \simeq 300 \mathrm{TeV}$. Such a scenario is easy to obtain in our instanton-mediated model: $\Lambda_{n \psi}^{4}=\left[e^{+S_{E 2}} M_{S}\right]^{2} m_{\tilde{g}}^{2}$ so that $e^{+S_{E 2}} M_{S} \simeq m_{\tilde{g}} \simeq 300 \mathrm{TeV}$ as well as $m_{\tilde{g}} \simeq 1 \mathrm{TeV}$ and $e^{+S_{E 2}} M_{S} \simeq 10^{5} \mathrm{TeV}$ can be interesting. In both cases, $M_{S}$ is much smaller than the Planck scale. Let us comment that if $M_{S} \simeq$ $100 \div 1000 \mathrm{TeV}$, the hierarchy problem of the Higgs mass will be alleviated from $m_{H}^{2} / 10^{38} \mathrm{GeV}^{2} \simeq 10^{-34}$ to $m_{H}^{2} / 10^{4 \div 6} \mathrm{TeV}^{2} \simeq 10^{-6} \div 10^{-8}$. As a consequence, a possible future detection of $n-\bar{n}$-transition eventually may suggest a future plan for $100 \div 1000 \mathrm{TeV}$ protonproton colliders after LHC. On the other hand, because of $e^{S_{E 2}}$-factor, $M_{S}=10 \mathrm{TeV}$ remains compatible with our space of parameters: LHC will immediately test this scenario in the next run. Another possible case can be $\Lambda_{n \psi} \simeq 10^{12 \div 13} \mathrm{TeV}$ while $\Lambda_{C P T V} \simeq 100 \div 10^{3} \mathrm{GeV}$. In this case, $e^{+S_{E 2}} M_{S} \simeq m_{\tilde{g}} \simeq 10^{12 \div 13} \mathrm{TeV}$.

Now, let us discuss another plausible scenario: $m_{\psi} \simeq$ $\mu_{\psi}$. In this case, $\mu_{n \bar{n}} \simeq \mu_{n \psi}^{2} \mu_{\psi} / \Lambda_{C P T V}^{2}$. Region of the parameters are practically the same discussed later, replacing $3 \Lambda_{C P T V} \rightarrow \Lambda_{C P T V}$. But in this case, $n-\bar{n}$ is entirely generated by $\Lambda_{C P T V}$. On the other hand, $\left|\Delta m_{n \bar{n}}\right| \simeq 2 \mu_{\psi n}^{2} / \Lambda_{C P V}$, so that $\mu_{n \bar{n}} \simeq\left|\Delta m_{n \bar{n}}\right| / 2$. In this case, actual limits on $\Delta m_{n \bar{n}}$ are roughly $10^{-23} \mathrm{eV}$ 33 .

\section{CONCLUSIONS AND REMARKS}

In this paper, we have shown a mechanism for a dynamical CPTV, mediated from a hidden sector to the neutron (antineutron) one. In particular, a sterile partner of the neutron $\psi$ can be introduced. We have called such a particle "neutronic weight": it is a weight for our desired CPTV see-saw. An effective neutron-nweight vertex transmits informations about the hidden CPTV to the neutron sector. As shown, such a vertex can be induced by exotic instantons, without provoking other dangerous ones. As a consequence, a CPTV mass term for the neutron is generated. We remark that such a mass term is naturally small, but testable in the next generation of experiments in $n-\bar{n}$-transitions! On the other hand, such mechanism can be related to a primordial CPTV baryogenesis through $\psi$-decays $\psi \rightarrow u d d, \bar{u} \bar{d} \bar{d}$ 
or in three quarks collisions $u d d \rightarrow \bar{u} \bar{d} \bar{d}$. In this case, Sakharov's conditions are not satisfied and Baryonviolations can be also generated in thermal equilibrium. See [18 for discussions of these aspects in other contests and in generic CPTV models.

On the other hand, our model suggests a direct test in future colliders. In fact, exotic instantons can be resonantly produced in $100-1000 \mathrm{TeV}$ collisions like $u d d, u d s, u b s, . ., \rightarrow \psi$. In this case, a distinct 'cutoff' in correspondent cross sections, related to effective lenght scale of the exotic instanton, can provide a hint against other gauge models.

For these motivations, we conclude that so mild limits on neutron physics could hide unexpected CPTV effects, despite of theoretical prejudices: only experimental ob- servables will have the "last word" about it.

\section{Acknowledgments}

I would like to thank my collaborators Zurab Berezhiani and Massimo Bianchi for interesting conversations on these subjects. I also would like to thank Parsa Ghorbani, Sergei Gninenko, Alan Kostelecky, Xianwei Kang and Augusto Sagnotti for interesting and useful comments.

My work was supported in part by the MIUR research grant "Theoretical Astroparticle Physics" PRIN 2012CPPYP7.
[1] L. B. Okun, hep-ph/0210052 L. B. Okun, hepph/9612247 V.A. Kostelecky, Perspectives on Lorentz and CPT Violation, Presented at 4th Meeting on CPT and Lorentz Symmetry, Bloomington, Indiana, 8-11 Aug 2007; e-Print: arXiv:0802.0581 [gr-qc]; V.A. Kostelecky and S. Samuel, Phys. Rev. Lett. 63224 (1989); D. Colladay and V. A. Kost- elecky, Phys. Rev. D 58, 116002 (1998) hep-ph/9809521. S. R. Coleman and S. L. Glashow, Phys. Rev. D 59, 116008 (1999) hep-ph/9812418; J. R. Ellis, N. E. Mavromatos, D. V. Nanopoulos, A. S. Sakharov and E. K. G. Sarkisyan, Astropart. Phys. 25, 402 (2006) [Astropart. Phys. 29, 158 (2008) astro-ph/0510172; T. Jacobson, S. Liberati and D. Mattingly, Annals Phys. 321, 150 (2006); F. Allmendinger, W. Heil, S. Karpuk, W. Kilian, A. Scharth, U. Schmidt, A. Schnabel and Y. Sobolev et al., Phys. Rev. Lett. 112, no. 11, 110801 (2014) arXiv:1312.3225 [gr-qc]]; Y. V. Stadnik and V. V. Flambaum, Eur. Phys. J. C 75, no. 3, 110 (2015) arXiv:1408.2184[hep-ph]]; V. A. Kostelecky and N. Russell, Rev. Mod. Phys. 83, 11 (2011) arXiv:0801.0287 [hep-ph]]; V. A. Kostelecky and M. Mewes, Phys. Rev. D 69, 016005 (2004) hep-ph/0309025; V. A. Kostelecky, Phys. Rev. Lett. 80, 1818 (1998) hep-ph/9809572; V. A. Kostelecky and M. Mewes, Phys. Rev. D 66, 056005 (2002) hep-ph/0205211; V. A. Kostelecky, Phys. Rev. D 64, 076001 (2001) hep-ph/0104120; V. A. Kostelecky and R. Lehnert, Phys. Rev. D 63, 065008 (2001) hep-th/0012060; A. Kostelecky and M. Mewes, Phys. Rev. D 85, 096005 (2012) arXiv:1112.6395 [hep-ph]]; M. Chaichian, A. D. Dolgov, V. A. Novikov and A. Tureanu, Phys. Lett. B 699 (2011) 177 arXiv:1103.0168 [hep-th]]; N. E. Mavromatos, Hyperfine Interact. 228 (2014) 1-3, 7 arXiv:1312.4304 [hep-ph]]; N. E. Mavromatos, Found. Phys. 40 (2010) 917 arXiv:0906.2712 [hep-th]]; N. E. Mavromatos, J. Phys. Conf. Ser. 171 (2009) 012007 arXiv:0904.0606 [hep-ph]]; N. E. Mavromatos, PoS KAON (2008) 041 arXiv:0707.3422 [hepph]]; J. Bernabeu, J. R. Ellis, N. E. Mavromatos, D. V. Nanopoulos and J. Papavassiliou, In *Di Domenico, A. (ed.): Handbook on neutral kaon interferometry at a Phi-factory* 39-83 hep-ph/0607322; N. E. Mavromatos, AIP Conf. Proc. 796 (2005) 13 hep-ph/0506294];
C. A. Escobar and M. A. G. Garcia, arXiv:1505.00069 [hep-th]; T. D. Gutierrez, arXiv:1504.01417 [hep-th]; M. Randhawa, M. Singh and M. Gupta, Phys. Rev. D 91 (2015) 1, 017301 arXiv:1412.4903 [hep-ph]]; K. Fujikawa and A. Tureanu, Phys. Lett. B $\mathbf{7 4 3}$ (2015) 39 arXiv:1409.8023 [hep-ph]]; Y. F. Li and Z. h. Zhao, Phys. Rev. D 90 (2014) 11, 113014; H. Belich, L. D. Bernald, P. Gaete, J. A. Helayl-Neto and F. J. L. Leal, arXiv:1502.06126 [hep-th]; Y. V. Stadnik, B. M. Roberts and V. V. Flambaum, Phys. Rev. D 90 (2014) 045035 arXiv:1407.5728 [hep-ph]]; S. Y. Li, J. Q. Xia, M. Li, H. Li and X. Zhang, Astrophys. J. 799 (2015) 2, 211 arXiv:1405.5637 [astro-ph.CO]]; T. de Paula Netto and I. L. Shapiro, Phys. Rev. D 89 (2014) 10, 104037 arXiv:1403.3152 [hep-th]]; Y. V. Stadnik and V. V. Flambaum, Eur. Phys. J. C 75 (2015) 3, 110 arXiv:1408.2184 [hep-ph]]; T. R. S. Santos and R. F. Sobreiro, arXiv:1502.06881 [hep-th].

[2] R. Adler et al. [CPLEAR Collaboration], Nucl. Phys. Proc. Suppl. 59 (1997) 182.

J. Ellis et al., Phys. Lett. B 364 (1995) 239.

M. Silarski [KLOE-2 Collaboration], J. Phys. Conf. Ser. 556 (2014) 1, 012071.

[3] M. Baldo-Ceolin et al., Z. Phys. C 63, 409 (1994).

[4] K. Babu, S. Banerjee, D. V. Baxter, Z. Berezhiani, M. Bergevin, S. Bhattacharya, S. Brice and T. W. Burgess et al., arXiv:1310.8593 [hep-ex]; D. G. Phillips, II, W. M. Snow, K. Babu, S. Banerjee, D. V. Baxter, Z. Berezhiani, M. Bergevin and S. Bhattacharya et al., arXiv:1410.1100 [hep-ex]].

[5] Yu. Abov, F. Dzheparov, L.B. Okun, JETP Lett. 39, 493 (1984).

[6] K. S. Babu and R. N. Mohapatra, arXiv:1504.01176 [hep$\mathrm{ph}]$.

[7] L. E. Ibanez and A. M. Uranga, JHEP 0703 (2007) 052 hep-th/0609213; L. E. Ibanez, A. N. Schellekens and A. M. Uranga, JHEP 0706 (2007) 011 arXiv:0704.1079 [hep-th]]; L. E. Ibanez and A. M. Uranga, String theory and particle physics: An introduction to string phenomenology, Cambridge, UK: Univ. Pr. (2012) 673 p.; R. Blumenhagen, M. Cvetic, D. Lust, R. Richter and T. Weigand, Phys. Rev. Lett. 100 (2008) 061602 arXiv:0707.1871 [hep-th]]; R. Blumenhagen, M. Cvetic 
and T. Weigand, Nucl. Phys. B 771 (2007) 113 hep-th/0609191; B. Florea, S. Kachru, J. McGreevy, N. Saulina, JHEP 0705 (2007) 024. hep-th/0610003; M. Cvetic, J. Halverson, P. Langacker and R. Richter, JHEP 1010 (2010) 094 arXiv:1001.3148 [hep-th]]; M. Cvetic, T. Weigand, Phys. Rev. Lett. 100 (2008) 251601. arXiv:0711.0209 [hep-th]]; M. Cvetic, R. Richter, T.Weigand, Phys. Rev. D76 (2007) 086002. hepth/0703028; Y. Hamada, T. Kobayashi and S. Uemura, JHEP 1405 (2014) 116 arXiv:1402.2052 [hepth]]; H. Abe, T. Kobayashi, Y. Tatsuta and S. Uemura, arXiv:1502.03582 [hep-ph].

[8] A. Addazi and M. Bianchi, JHEP 1412 (2014) 089 arXiv:1407.2897 [hep-ph]]; A. Addazi, arXiv:1501.04660 hep-ph]; A. Addazi and M. Bianchi, arXiv:1502.01531 [hep-ph]; A. Addazi and M. Bianchi, arXiv:1502.08041 [hep-ph]; A. Addazi, arXiv:1504.06799 [hep-ph]; A. Addazi, arXiv:1505.00625 [hep-ph]; A. Addazi, arXiv:1506.06351 [hep-ph].

[9] L. E. Ibanez, F. Marchesano and R. Rabadan, JHEP 0111, 002 (2001), hep-th/0105155 R. Blumenhagen, B. Kors, D. Lust and T. Ott, Nucl. Phys. B 616, 3 (2001), hep-th/0107138; M. Cvetic, G. Shiu and A. M. Uranga, Phys. Rev. Lett. 87, 201801 (2001), hepth/0107143 D. Bailin, G. V. Kraniotis and A. Love, Phys. Lett. B 530, 202 (2002), hep-th/0108131. D. Cremades, L. E. Ibanez and F. Marchesano, JHEP 0207, 009 (2002), hep-th/0201205, D. Cremades, L. E. Ibanez and F. Marchesano, JHEP 0207, 022 (2002), hepth/0203160 L. E. Ibanez, hep-ph/0109082 R. Blumenhagen, V. Braun, B. Kors and D. Lust, hep-th/0210083. D. Cremades, L. E. Ibanez and F. Marchesano, hepph/0212048, D. Lust, Class. Quant. Grav. 21 (2004) S1399 hep-th/0401156; G. Aldazabal, L. E. Ibanez, F. Quevedo, and A. M. Uranga, JHEP 08 (2000) 002, arXiv:hep-th/0005067; D. Berenstein, V. Jejjala, and R. G. Leigh, Phys.Rev.Lett. 88 (2002) 071602, arXiv:hepph/0105042 [hep-ph]; G. Aldazabal, L. E. Ibanez, and F. Quevedo, JHEP 0002 (2000) 015, arXiv:hep-ph/0001083 [hep-ph]; J. Cascales, M. Garcia del Moral, F. Quevedo, and A. Uranga, JHEP 0402 (2004) 031, arXiv:hepth/0312051 [hep-th].

[10] G. Aldazabal, S. Franco, L.E. Ibanez, R.Rabadan, and A. M. Uranga, J. Math. Phys. 42 (2001) 3103-3126, hepth/0011073 M. Cvetic, J. Halverson and R. Richter, JHEP 0912 (2009) 063 arXiv:0905.3379 [hep-th]].

[11] Y.A.Kamyshkov, "BLV-2011 Workshop, September 2224, 2011", http://utkhep.phys.utk.edu/BLV/DocDB /0000/000076/001/Addazi.pdf; A. Addazi, Nuovo Cim. C 038 (2015) 01, 21; A. Addazi, Z.Berezhiani, Y.A.Kamyshkov, in preparation.

[12] S. N. Gninenko, Phys. Rev. D 91 (2015) 1, 015004 arXiv:1409.2288 [hep-ph]]; S. N. Gninenko and N. V. Krasnikov, arXiv:1503.01595 [hep-ph].

[13] V. A. Kostelecky and R. Lehnert, Phys. Rev. D 63 (2001) 065008 hep-th/0012060.

[14] A. Addazi and S. Capozziello, Int.J.Theor.Phys. 54 (2015) 6, 1818-1829; arXiv:1407.4840 [gr-qc].

[15] A. Addazi and G. Esposito, arXiv:1502.01471 [hep-th].

[16] A. Addazi, arXiv:1505.07357[hep-th].

[17] Z. Berezhiani and L. Bento, Phys. Rev. Lett. 96 (2006) 081801 hep-ph/0507031.

[18] A.D. Dolgov, Ya.B. Zeldovich, Uspekhi Fiz. Nauk, 130, 559 (1980); Rev. Mod. Phys. 531 (1981); Berto- lami O, Colladay D, Kostelecky V A and Potting R 1997 Phys.Lett. B395 178183 (Preprint hep-ph/9612437); A. D. Dolgov, Phys. Atom. Nucl. 73 (2010) 588 arXiv:0903.4318 [hep-ph]]; G. Barenboim, N.E. Mavromatos, Phys. Rev. D70, 093015 (2004); hep-ph/0406035 J. Ellis, N. E. Mavromatos and S. Sarkar, Phys. Lett. B 725 (2013) 407 arXiv:1304.5433 [gr-qc]]; N. E. Mavromatos and S. Sarkar, Eur. Phys. J. C 73 (2013) 3, 2359 arXiv:1211.0968 [hep-ph]].

[19] A. Sagnotti, IN *CARGESE 1987, PROCEEDINGS, NONPERTURBATIVE QUANTUM FIELD THEORY* 521-528 AND ROME II UNIV. - ROM2F-87-025 (87,REC.MAR.88) 12p hep-th/0208020; A. Sagnotti, Phys. Lett. B294 (1992) 196 hep-th/9210127; C. Angelantonj and A. Sagnotti, Phys. Rept. 1 (Erratum-ibid.) 339] arXiv:hep-th/0204089, G. Pradisi and A. Sagnotti, Phys. Lett. B 216 (1989) 59; M. Bianchi and A. Sagnotti, Phys. Lett. B247 (1990) 517; M. Bianchi, G. Pradisi and A. Sagnotti, Nucl. Phys. B376 (1992) 365; D. Fioravanti, G. Pradisi and A. Sagnotti, Phys. Lett. B321 (1994) 349 arXiv:hep-th/9311183; A. Sagnotti, in *Palaiseau 1995, Susy 95* 473-484 hep-th/9509080]; A. Sagnotti, Nucl. Phys. Proc. Suppl. 56B (1997) 332 arXiv:hepth/9702093; C. Angelantonj, M. Bianchi, G. Pradisi, A. Sagnotti, and Y. Stanev, Phys.Lett. B385 (1996) 96-102, arXiv:hep-th/9606169 [hep-th].

[20] H. Ghorbani, D. Musso and A. Lerda, JHEP 1103 (2011) 052 arXiv:1012.1122 [hep-th]]; H. Ghorbani and D. Musso, JHEP 1112 (2011) 070 arXiv:1111.0842 [hep-th]]; H. Ghorbani, JHEP $\mathbf{1 3 1 2}$ (2013) 041 arXiv:1306.1487 [hep-th]].

[21] A. Addazi, Z. Berezhiani, R. Bernabei, P. Belli, F. Cappella, R. Cerulli and A. Incicchitti, arXiv:1507.04317 [hep-ex].

[22] X. W. Kang, H. B. Li and G. R. Lu, Phys. Rev. D 81 (2010) 051901 arXiv:0906.0230 [hep-ph]]; X. W. Kang, H. B. Li and G. R. Lu, arXiv:1008.2845 [hep-ph].

[23] A. Addazi, arXiv:1508.04054 [gr-qc].

[24] To Break Lorentz Invariance can be problematic also at classical level. For an interesting explicit example, in [14] geodetic instabilities of Classical Lorentz Breaking Massive Gravity were discussed. A discussion of these effects is often neglected in literature of Modified Gravities and Massive gravities. On the other hand, issues about problematic acausal divergences at quantum level for Non-local QFTs were discussed in [15]. In this last case, $\mathcal{N}=1$ supersymmetry seems to eliminate many acausal diagrams, but not other many ones, as carefully checked in [15]. Probably, $\mathcal{N}=2$ susy can ulteriorly alleviate acausal divergences. In [16, how the formation of classicalons can avoid acausal divergences in scattering amplitudes is discussed.

[25] The first proposal of a see-saw type I mechanism for the neutron (CPT-preserving) was shown by Berezhiani et al in [17, for neutron-mirror neutron oscillations. Clearly, Berezhiani's diagram can be considered also for $n-\bar{n}$. In this diagram, $\psi$ is substituted by a sort of $\mathrm{RH}$ neutron, with baryon number $B=-1$ and a B-violating Majorana mass. In this model, they assume that other possible gauge invariant interactions of the $\mathrm{RH}$ neutrons with SM particles are not allowed. Considerations done in this paper can also be applied for this model. This model could be fully justified by appropriate extra discrete symmetries or by exotic instantons in non-susy intersecting 
D-branes' models.

[26] Because of this, signals in neutron physics have to be dependent on the momentum of neutrons (antineutrons). However, in our paper, we are interested to low energy observables, so that momentum-dependent effects are completely negligible. On the other hand, in the opposite regime, momentum dependent effects in neutron physics would afflict neutron(antineutron) propagation. Unfortunately, it is not simple to try an interesting example in phenomenology in which such a test could be realistically done. With the increasing of statistics in UHECR, one could limit such Lorentz/CPT violations in future, if a consistent part of UHECR was composed of protons.

[27] In principle, one could introduce other possible Lorentz violating couplings for $\psi$ such as $b_{\mu} \bar{\psi} \gamma^{\mu} \gamma_{5} \psi$ (with $b_{\mu}$ a spurion field getting a LIV expectation value) and so on, as usually proposed in literature mentioned above. These other possible operators would affect neutron (antineutron) physics through our see-saw mechanism. In this paper, we have focalized on the case in which the only relevant LIV OPE is $a_{\mu} \bar{\psi} \gamma^{\mu} \psi$. A more general case will deserve future investigations beyond the purposes of this paper.

[28] Let us remind some useful seminal papers on open string theories in [19.

[29] However, there are other classes of instantons that may be relevant for our purposes. These classes were studied in [20]. I would like to thank Parsa Ghorbani for discussions on these aspects.

[30] $G$ naturally could be a parallel intersecting D-brane world. This can intriguing connections with Asymmetric Mirror dark matter phenomenology in direct detection 21.

[31] The author of this paper retains more probable such a mechanism for a CPT breaking with respect to deco- herence quantum gravity effects from virtual miniblack holes, violating quantum mechanics principles. See 23 for related discussions on these aspects.

[32] Another comment can be that such a scenario just seems to displace the fine-tuning of a CPTV mass for the neutron to the fine-tuning of a so small CPTV mass for the nweight. But regardless of our aesthetic prejudices, we have analyzed these cases as a possible region of the parameter space.

[33] Let us note that the superpotential generated by exotic instantons 13 has a Yukawa matrix with respect to flavors' indices. This operator can also induce $\Lambda^{0}-\bar{\Lambda}^{0}$ transitions, violating $B$ as $\Delta B=2$. As a consequence, our model inevitably predicts the propagation of CPTV phases also in this channel. However, mixed disk amplitudes considered are generically not democratic with flavors. As a consequence, CPTV phases' ratio of $\Lambda^{0}-\bar{\Lambda}^{0}$ and $n-\bar{n}$ has an extra parameter depending on the particular homologies in mixed disk amplitudes considered. This can have relevant implications in $\Lambda^{0}-\bar{\Lambda}^{0}$ physics. Possibilities to constrains CPTV phases in $\Lambda^{0}-\bar{\Lambda}^{0}$ sector were proposed in 22]: our model can be considered as a theoretical framework motivating these phenomenological discussions. However, as mentioned above, one has also to consider energy dependences of CPTV phases. Probably, these dependences can be also neglected in these channels, but these aspects will deserve future investigations beyond the purposes of this short paper. On the other hand, strong indirect constrains related to this $\Delta B=2$ channel are coming from dinucleon decays $N N \rightarrow K K: 100 \mathrm{TeV}$ on the new physics scale, against $100 \mathrm{GeV}$ from electron-positron colliders. I am grateful to Xianwei Kang for useful informations and remarks in these subjects. 